\title{
Ultra-Long Pharmacokinetic Properties of Insulin Degludec are Comparable in Elderly Subjects and Younger Adults with Type 1 Diabetes Mellitus
}

\author{
S. Korsatko $\cdot$ S. Deller $\cdot$ J. K. Mader $\cdot$ K. Glettler • \\ G. Koehler • G. Treiber • M. Urschitz $\cdot$ M. Wolf • \\ H. Hastrup · F. Søndergaard $\cdot$ H. Haahr $\cdot$ T. R. Pieber
}

Published online: 22 November 2013

(c) The Author(s) 2013. This article is published with open access at Springerlink.com

\begin{abstract}
Background Management of diabetes in elderly subjects is complex and careful management of glucose levels is of particular importance in this population because of an increased risk of diabetes-related complications and hypoglycaemia.

Objective The aim of this study was to evaluate the pharmacokinetic and pharmacodynamic properties of insulin degludec (IDeg), a basal insulin with an ultra-long duration of action, in elderly subjects with type 1 diabetes compared with younger adults.

Methods This trial was a randomised, double-blind, twoperiod, crossover trial conducted in a single centre and included both inpatient and outpatient periods. Subjects were men and women aged 18-35 years inclusive (younger adult group) or $\geq 65$ years (elderly group) with type 1 diabetes who received IDeg $(0.4 \mathrm{U} / \mathrm{kg})$ via subcutaneous injection in the thigh once-daily for six days. Following 6-day dosing, a 26-hour euglycaemic glucose clamp procedure was conducted to evaluate the steady-state
\end{abstract}

S. Korsatko $\cdot$ J. K. Mader $\cdot$ K. Glettler $\cdot$ G. Koehler

G. Treiber · M. Urschitz · M. Wolf · T. R. Pieber $(\square)$

Division of Endocrinology and Metabolism, Department of

Internal Medicine, Medical University of Graz,

Auenbruggerplatz 15, $8036 \mathrm{Graz}$, Austria

e-mail: thomas.pieber@medunigraz.at

S. Deller

Center for Medical Research, Medical University of Graz, Graz, Austria

H. Hastrup · H. Haahr

Novo Nordisk A/S, Søborg, Denmark

F. Søndergaard

Novo Nordisk A/S, Aalborg, Denmark pharmacodynamic effects of IDeg. Blood samples were taken for pharmacokinetic analysis up to $120 \mathrm{~h}$ post-dose. Pharmacokinetic endpoints included the total exposure of IDeg, ie the area under the IDeg serum concentration curve during one dosing interval at steady state $\left(\mathrm{AUC}_{\mathrm{IDeg}, \tau, \mathrm{SS}}\right)$ ( $\tau=0-24 \mathrm{~h}$, equal to one dosing interval) and the maximum IDeg serum concentration at steady state $\left(\mathrm{C}_{\mathrm{max}, \text { IDeg,Ss }}\right)$. Pharmacodynamic endpoints included the total glucoselowering effect of IDeg, ie the area under the glucose infusion rate (GIR) curve at steady state $\left(\mathrm{AUC}_{\mathrm{GIR}, \tau, \mathrm{SS}}\right)$, and the maximum GIR at steady state ( $\left.\mathrm{GIR}_{\text {max,IDeg,SS }}\right)$.

Results Total exposure ( $\left.\mathrm{AUC}_{\mathrm{IDeg}, \tau, \mathrm{SS}}\right)$ and maximum concentration $\left(\mathrm{C}_{\mathrm{max}, \text { IDeg,Ss }}\right)$ of IDeg were comparable between elderly subjects and younger adults. Estimated mean age group ratios (elderly/younger adult) for $\mathrm{AUC}_{\mathrm{IDeg}, \tau, \mathrm{SS}}$ and $\mathrm{C}_{\text {max,IDeg,Ss }}$ and corresponding two-sided $95 \%$ confidence intervals (CIs) were 1.04 (95\% CI 0.73-1.47) and 1.02 (95\% CI 0.74-1.39), respectively. Mean $\mathrm{AUC}_{\mathrm{IDeg}, 0-12 \mathrm{~h}, \mathrm{SS}} / \mathrm{AUC}_{\mathrm{IDeg}, \tau, \mathrm{SS}}$ was $53 \%$ in both younger adult and elderly subjects, showing that in both age groups IDeg exposure was evenly distributed across the first and second $12 \mathrm{~h}$ of the 24-hour dosing interval. No statistically significant differences were observed between younger adult and elderly subjects with regard to $\mathrm{AUC}_{\mathrm{GIR}, \tau, \mathrm{SS}}$ (the primary endpoint of this study) and

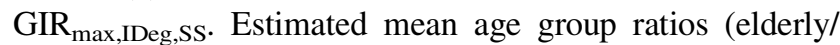
younger adult) for $\mathrm{AUC}_{\mathrm{GIR}, \tau, \mathrm{SS}}$ and $\mathrm{GIR}_{\text {max,IDeg,SS }}$ and corresponding two-sided $95 \%$ CIs were 0.78 (95\% CI 0.47-1.31) and 0.80 (95\% CI 0.54-1.17), respectively. Duration of action was beyond the clamp duration of $26 \mathrm{~h}$ in all subjects.

Conclusions The exposure of IDeg at steady state during once-daily dosing was similar in younger adult and elderly subjects. The glucose-lowering effect of IDeg was numerically lower in elderly subjects compared with younger adults, but no significant differences were 
observed between age groups. The ultra-long pharmacokinetic and pharmacodynamic properties of IDeg observed in younger adults were preserved in elderly subjects with type 1 diabetes.

Clinical trials.gov number: NCT00964418

\section{Introduction}

Management of diabetes mellitus in elderly subjects is complex. This heterogeneous patient population includes individuals with diabetes who have few or no co-morbidities, as well as others with additional chronic disorders, differing degrees of diabetes-related co-morbidities or cognitive impairment, and the physically frail [1]. Central to effective diabetes management is ensuring optimal control of blood glucose levels. This is of particular importance in elderly subjects, who carry an increased risk of developing long-term diabetes-related complications [1]. Furthermore, elderly subjects with advanced co-morbidities, polypharmacy and existing, advanced diabetes-related complications are at an increased risk of insulin-induced hypoglycaemia, associated with higher levels of morbidity and mortality $[2,3]$. This increased susceptibility is exacerbated by long-standing or established disease and hypoglycaemic unawareness, with individuals even more vulnerable if living alone [4].

Insulin degludec (IDeg) is a new-generation basal insulin developed for once-daily administration, which has a distinct absorption mechanism. Upon subcutaneous (SC) injection, IDeg forms long chains of multi-hexamers, resulting in a soluble depot in the SC tissue from which IDeg monomers gradually separate. This mechanism provides a slow and continuous absorption of IDeg into the circulation, leading to flat, stable and ultra-long pharmacokinetic and pharmacodynamic profiles [5]. Moreover, IDeg has four times less within-subject variability in glucose-lowering effect compared with insulin glargine (IGlar) [6]. This low level of variability in glucose-lowering effect suggests that IDeg may have utility in treating elderly subjects, where the avoidance of hypoglycaemia is of increased clinical importance.

Treatment of elderly subjects with diabetes is further confounded by the limited clinical data currently available in this subject population regarding the use of long-acting insulin [2]. Therefore, studies evaluating the effects of long-acting insulin in elderly subjects via a euglycaemic clamp procedure would represent a valuable addition to the clinical literature.

The primary objective of the present study was to compare the pharmacokinetic and pharmacodynamic properties of IDeg between elderly subjects and younger adults with type 1 diabetes. Steady-state pharmacodynamic properties of IDeg in elderly and younger adult subjects were evaluated using a $26-\mathrm{h}$ euglycaemic clamp procedure.

\section{Materials and Methods}

\subsection{Study Design}

This study was a single-centre (Department of Internal Medicine, Division of Endocrinology and Metabolism, Medical University of Graz, Austria), randomised, multiple-dose, double-blind, two-period, crossover trial conducted in subjects with type 1 diabetes (Clinical trials.gov number: NCT00964418). Before initiation, the trial was reviewed and approved by the local independent ethics committee according to local regulations. The trial was performed in accordance with the Declaration of Helsinki and its amendments in force at the initiation of the trial. Subjects were informed of the risks and benefits of the trial and that they could withdraw at any time for any reason. Consent was obtained in writing before any trialrelated activities, and the investigator retained the consent forms.

\subsection{Subjects}

Study participants were men and women aged 18-35 years inclusive (younger adult group) or aged $\geq 65$ years (elderly group) with type 1 diabetes for $\geq 12$ months, and a fasting C-peptide level below $0.3 \mathrm{nmol} / \mathrm{L}$. Other key inclusion criteria for the trial included subjects who had been treated with multiple daily insulin injections (or continuous SC insulin infusion) for $\geq 12$ months; a body mass index (BMI) of $18.0-28.0 \mathrm{~kg} / \mathrm{m}^{2}$ inclusive; a daily basal insulin requirement of $\geq 0.2 \mathrm{IU} / \mathrm{kg} / \mathrm{day}$; a total daily insulin treatment of $<1.2 \mathrm{IU} / \mathrm{kg} / \mathrm{day}$; and a glycosylated haemoglobin level of $\leq 10.0 \%$.

Exclusion criteria for study participation included donation of blood or plasma in the past month or donations of $>500 \mathrm{~mL}$ within 3 months before screening; use of systemic [oral or intravenous (IV)] corticosteroids, monoamine oxidase inhibitors, non-selective beta-blockers, growth hormone, non-routine vitamins or herbal products; use of thyroid hormones, unless this use had been stable during the previous 3 months; smoking or the use of nicotine gum or transdermal nicotine patches during the inpatient period; recurrent severe hypoglycaemia (more than one severe hypoglycaemic event during the last 12 months); hypoglycaemic unawareness as judged by the investigator; and hospitalisation for diabetic ketoacidosis during the previous 6 months. 


\subsection{Interventions and Pharmacokinetic Sampling}

Following screening (Visit 1), subjects were randomly allocated 1:1 to one of two predetermined treatment sequences, each comprising two 6-day treatment periods (Period 1, Visits 2-8; Period 2, Visits 9-15). Each treatment period included once-daily SC dosing with $0.4 \mathrm{U} / \mathrm{kg}$ of IDeg or IGlar, in a two-period crossover design. IGlar was included primarily as a control in case differences between age groups were observed for IDeg. As this was not the case, only results from IDeg treatment are reported herein. The two treatment periods were separated by a washout period of 7-21 days, during which time subjects resumed their normal insulin treatment.

IDeg [provided in 3-mL Penfill ${ }^{\circledR}$ cartridges $(100 \mathrm{U} / \mathrm{mL})$ ] and IGlar [provided in 3-mL cartridges $(100 \mathrm{U} / \mathrm{mL})$ ] were administered once daily at approximately $8.00 \mathrm{pm}$ via SC injection into a lifted skin fold on the anterior surface of the thigh using a syringe and needle. This region of injection is commonly used for the injection of basal or long-acting insulin preparations because of the slower absorption from this site [7]. Doses were prepared by a study nurse not otherwise involved in the trial, to maintain study blinding. During treatment periods, additional control of blood glucose levels was accomplished by bolus injections of insulin aspart, which were administered into a lifted skin fold on the lower abdominal wall and were supervised by the investigator on a daily basis. As a general guidance to serve as a basis for the supervision of the bolus insulin doses, the daily mean pre-prandial plasma glucose values were to be below or equal to $8.0 \mathrm{mmol} / \mathrm{L}(144 \mathrm{mg} / \mathrm{dL})$. The last injection of insulin aspart was no later than $10 \mathrm{~h}$ before dosing on each clamp visit.

Initial dosing in each treatment period took place during Visit 2 (Period 1) and Visit 9 (Period 2), respectively. During these 48-h in-house visits, the first three once-daily doses of IDeg or IGlar were administered at time points 0 , 24 and $48 \mathrm{~h}$. Subjects subsequently attended the clinic daily for two further outpatient visits (Visits 3 and 4, and Visits 10 and 11). Blood samples for assessment of pharmacokinetic parameters were taken before the first dosing, at frequent intervals within the first $24 \mathrm{~h}$ after the first dosing (at intervals of $0.25-2 \mathrm{~h}$ ) and immediately before subsequent doses (Visits 3 and 4, and Visits 10 and 11). The final dosing visit was an in-house visit (Visits 5 and 12) during which blood samples for steady-state pharmacokinetic assessment were taken regularly, both before (at hourly intervals from 1 to $5 \mathrm{~h}$ pre-dose and at $15 \mathrm{~min}$ predose) and for $48 \mathrm{~h}$ after dosing (at intervals of $0.5-10 \mathrm{~h}$ ). Blood samples were also taken for plasma glucose analysis every 5-30 min during this period up to $26 \mathrm{~h}$ post-dosing and at 28,38 and $48 \mathrm{~h}$ post-dosing. Subjects were discharged $48 \mathrm{~h}$ after final dosing, and returned at 72, 96 and
$120 \mathrm{~h}$ post-dose (Visits 6-8 and Visits $13-15$ ) for blood sampling for pharmacokinetic and plasma glucose analysis. A final follow-up visit (Visit 16) was conducted 7-21 days after the final dosing visit. The number of dosing days was chosen to ensure that all subjects reached steady state before a euglycaemic clamp was conducted.

\subsection{Euglycaemic Glucose Clamp Procedure}

The steady-state pharmacodynamic effects of IDeg were evaluated using a 26-h euglycaemic clamp procedure [target blood glucose $5.5 \mathrm{mmol} / \mathrm{L},(100 \mathrm{mg} / \mathrm{dL})$ ], beginning after final dosing, during Visit 5 (Period 1) and Visit 12 (Period 2). In brief, subjects fasted for at least $12 \mathrm{~h}$ before dosing and the start of the 26-h clamp, and remained fasted (with water ad libitum) and in a supine position until the clamp procedure was complete. Approximately $5 \mathrm{~h}$ before dosing a 3-h clamp run-in period was initiated, during which subjects received a variable IV infusion of human insulin (40 IU Actrapid ${ }^{\circledR}, 100 \mathrm{IU} / \mathrm{mL}$ in $99.6 \mathrm{~mL}$ saline) or glucose (10\%; Braun Infusomat FM, Melsungen, Germany) to obtain the glucose clamp target level of $5.5 \mathrm{mmol} / \mathrm{L}(100 \mathrm{mg} / \mathrm{dL})$. The target glucose clamp level was maintained during a 2 -h pre-dose clamp period leading up to the time of last dose administration. When IV insulin was used during the pre-dose clamp period, the rate of insulin infusion was decreased gradually post-dosing and terminated when glucose levels had declined by approximately $0.3 \mathrm{mmol} / \mathrm{L}$. A variable IV glucose infusion was then initiated to maintain the clamp target level [5.5 mmol/L, $(100 \mathrm{mg} / \mathrm{dL})]$. The clamp was maintained for $26 \mathrm{~h}$ after dosing; however, the clamp procedure was terminated early if plasma glucose levels consistently exceeded $11.1 \mathrm{mmol} / \mathrm{L}(200 \mathrm{mg} / \mathrm{dL})$ without any glucose infusion in the previous $30 \mathrm{~min}$.

\subsection{Data and Statistical Analyses}

The primary objective of the trial was to investigate the pharmacodynamic effects of IDeg at steady state in elderly subjects with type 1 diabetes versus younger adults with type 1 diabetes. Secondary objectives for the two age groups were to investigate the pharmacokinetic and pharmacodynamic profiles, including the pharmacokinetic exposure of IDeg at steady state, time to reach steady state and the safety of IDeg. Pharmacokinetic endpoints for IDeg included the area under the IDeg serum concentrationtime curve during one dosing interval at steady state $\left(\mathrm{AUC}_{\mathrm{IDeg}, \tau, \mathrm{SS}}\right)(\tau=0-24 \mathrm{~h})$, the maximum IDeg serum concentration at steady state $\left(C_{\max , \text { IDeg,SS }}\right)$ and the ratio between $\mathrm{AUC}_{\mathrm{IDeg}, 0-12 \mathrm{~h}, \mathrm{SS}}$ and $\mathrm{AUC}_{\mathrm{IDeg}, \tau, \mathrm{SS}} \quad\left(\mathrm{AUC}_{\mathrm{I}-}\right.$ Deg, $\left.0-12 \mathrm{~h}, \mathrm{SS} / \mathrm{AUC}_{\mathrm{IDeg}, \tau, \mathrm{SS}}\right)$. The primary pharmacodynamic endpoint for IDeg was the area under the glucose infusion 
rate (GIR) curve during one dosing interval at steady state $\left(\mathrm{AUC}_{\mathrm{GIR}, \tau, \mathrm{SS}}\right)(\tau=0-24 \mathrm{~h})$. Secondary pharmacodynamic endpoints included $\mathrm{GIR}_{\text {max,IDeg,SS, the ratio between }}$ $\mathrm{AUC}_{\mathrm{GIR}, 0-12 \mathrm{~h}, \mathrm{SS}}$ and $\mathrm{AUC}_{\mathrm{GIR}, \tau, \mathrm{SS}}\left(\mathrm{AUC}_{\mathrm{GIR}, 0-12 \mathrm{~h}, \mathrm{SS}} / \mathrm{AUC}-\right.$ $\mathrm{GIR}, \tau, \mathrm{SS}$ ), and the duration of action of IDeg (time from dose administration until the plasma glucose level increased to a level consistently above $8.3 \mathrm{mmol} / \mathrm{L}[150 \mathrm{mg} / \mathrm{dL}]$, during the glucose clamp procedure).

Safety assessments comprised adverse events (AEs), including local injection-site reactions, laboratory safety variables, physical examination, vital signs,

Table 1 Subject characteristics

\begin{tabular}{|c|c|c|}
\hline Characteristic & $\begin{array}{l}\text { Elderly } \\
\text { (aged } \geq 65 \text { years) }\end{array}$ & $\begin{array}{l}\text { Younger adult } \\
\text { (aged } 18-35 \text { years) }\end{array}$ \\
\hline Subjects, $n$ & 14 & 13 \\
\hline Mean (SD) age, years & $67.8(3.2)$ & $27.1(4.9)$ \\
\hline \multicolumn{3}{|l|}{ Sex, $n(\%)$} \\
\hline Female & $8(57)$ & $6(46)$ \\
\hline Male & $6(43)$ & $7(54)$ \\
\hline Mean (SD) BMI, $\mathrm{kg} / \mathrm{m}^{2}$ & $26.2(1.5)$ & $24.4(2.3)$ \\
\hline $\begin{array}{l}\text { Mean (SD) duration of } \\
\text { diabetes, years }\end{array}$ & $40.6(16.3)$ & $14.4(6.1)$ \\
\hline Mean (SD) $\mathrm{HbA}_{1 \mathrm{c}}, \%$ & $7.7(0.6)$ & $7.8(0.8)$ \\
\hline $\begin{array}{l}\text { Mean (SD) fasting } \\
\text { C-peptide, } \mathrm{nmol} / \mathrm{L}\end{array}$ & $0.03(0.07)$ & $0.01(0.01)$ \\
\hline Mean (SD) eGFR & $77.6(14.8)$ & $128.7(14.7)$ \\
\hline \multicolumn{3}{|l|}{$\begin{array}{l}\text { Insulin type at } \\
\text { screening, } n(\%)\end{array}$} \\
\hline Lispro & $2(14)$ & $7(54)$ \\
\hline Aspart & $11(79)$ & $3(23)$ \\
\hline Detemir & $3(21)$ & $2(15)$ \\
\hline Glargine & $7(50)$ & $3(23)$ \\
\hline Human insulin & $2(14)$ & $5(39)$ \\
\hline Isophane insulin (NPH) & $0(0)$ & $1(8)$ \\
\hline
\end{tabular}

$B M I$ body mass index, $e G F R$ estimated glomerular filtration rate, $H b A_{l c}$ glycosylated haemoglobin, $N P H$ neutral protamine Hagedorn, $S D$ standard deviation
Table 2 Pharmacokinetic endpoints for insulin degludec at steady state

\begin{tabular}{lll}
\hline Endpoint & $\begin{array}{l}\text { Elderly } \\
\text { (aged } \geq 65 \text { years })\end{array}$ & $\begin{array}{l}\text { Younger adult } \\
\text { (aged 18-35 years) }\end{array}$ \\
\hline AUC $_{\text {IDeg, } \tau, \mathrm{SS}, \mathrm{pmol} \cdot \mathrm{h} / \mathrm{L}}$ & 85,673 & 82,727 \\
$C_{\text {max,IDeg,Ss }}, \mathrm{pmol} / \mathrm{L}$ & 4481 & 4414 \\
$\mathrm{AUC}_{\mathrm{IDeg}, 0-12 \mathrm{~h}, \mathrm{SS}} /$ & $53(5)$ & $53(5)$ \\
$\mathrm{AUC}_{\mathrm{IDeg}, \tau, \mathrm{SS}}, \%(\mathrm{CV})$ & &
\end{tabular}

Elderly/younger adult $(95 \% \mathrm{CI})$

$$
\begin{array}{ll}
\mathrm{AUC}_{\mathrm{IDeg}, \tau, \mathrm{SS}} & 1.04(0.73-1.47) \\
C_{\text {max }, \mathrm{IDeg}, \mathrm{SS}} & 1.02(0.74-1.39)
\end{array}
$$

Mean values are estimated least square means, except for the mean ratio of $\mathrm{AUC}_{\mathrm{IDeg}, 0-12 \mathrm{~h}, \mathrm{SS}} / \mathrm{AUC}_{\mathrm{IDeg}, \tau, \mathrm{SS}}$, which was calculated and summarised using descriptive statistics

$A U C_{\text {IDeg, } \tau, S S}$ area under the insulin degludec serum concentration curve at steady state, $C V$ coefficient of variation, $C I$ confidence interval, $C_{\text {max } \text { IDeg,SS }}$ maximum insulin degludec serum concentration at steady state

electrocardiogram (ECG) and hypoglycaemic episodes (defined as 'confirmed' when they were either 'severe' as defined by the American Diabetes Association [8] or verified by a plasma glucose level of $<3.1 \mathrm{mmol} / \mathrm{L}$ [ $56 \mathrm{mg} / \mathrm{dL}]$ ).

IDeg serum concentrations were measured using a validated IDeg sandwich enzyme-linked immunosorbent assay. $\mathrm{AUC}_{\mathrm{IDeg}, 0-12 \mathrm{~h}, \mathrm{SS}}$ and $\mathrm{AUC}_{\mathrm{IDeg}, \tau, \mathrm{SS}}$ were calculated as the area under the insulin concentration-time profile using the linear trapezoidal method based on observed values and actual measurement times. Missing values were imputed using linear interpolation. $C_{\mathrm{max}, \mathrm{IDeg}, \mathrm{SS}}$ was derived from the individual concentration-time profiles.

$\mathrm{AUC}_{\mathrm{GIR}, \tau, \mathrm{SS}}$ was derived from individual GIR profiles and was calculated as the area under the smoothed GIR curve using the linear trapezoidal technique on interpolated points. GIR $_{\text {max,IDeg,ss }}$ was derived from the smoothed GIR curve.

All analyses were conducted on the full analysis set. Log-transformed $\mathrm{AUC}_{\mathrm{GIR}, \tau, \mathrm{SS}}, \mathrm{GIR}_{\text {max,IDeg,SS }}, \mathrm{AUC}_{\mathrm{IDeg}, \tau, \mathrm{SS}}$ and $C_{\text {max,IDeg,Ss }}$ values were analysed using an analysis of variance method with age group (elderly/younger adult)
Fig. 1 Mean pharmacokinetic profiles for insulin degludec at steady state

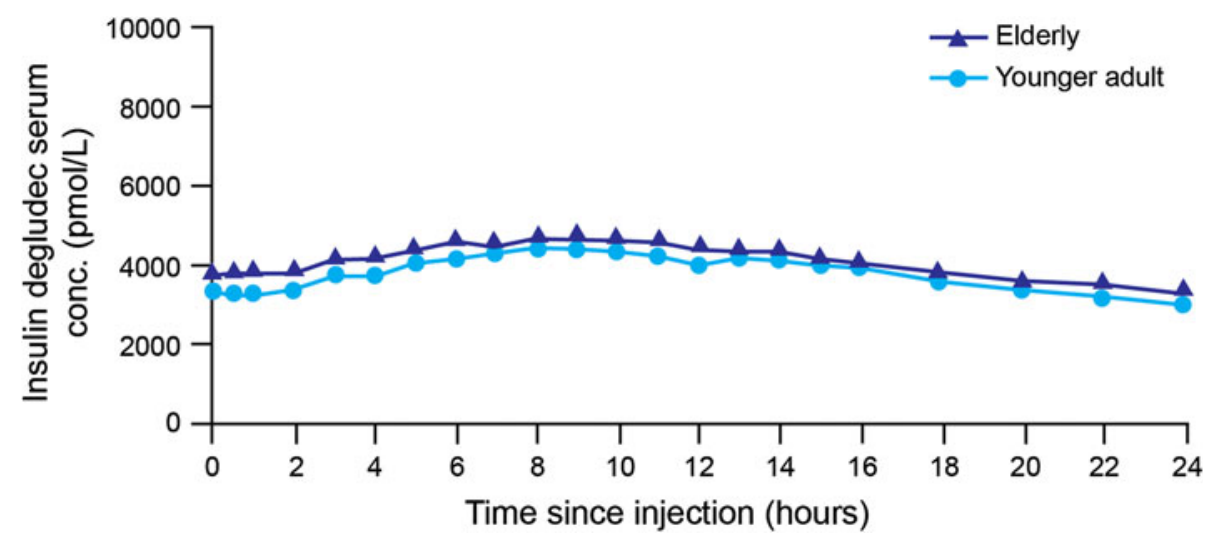


Table 3 Pharmacodynamic endpoints for insulin degludec at steady state

\begin{tabular}{|c|c|c|}
\hline Endpoint & $\begin{array}{l}\text { Elderly } \\
\text { (aged } \geq 65 \text { years) }\end{array}$ & $\begin{array}{l}\text { Younger adult } \\
\text { (aged 18-35 years) }\end{array}$ \\
\hline $\mathrm{AUC}_{\mathrm{GIR}, \tau, \mathrm{SS}}, \mathrm{mg} / \mathrm{kg}$ & 1,923 & 2,457 \\
\hline $\begin{array}{c}\mathrm{GIR}_{\text {max,IDeg,Ss, }} \\
\mathrm{mg} /(\mathrm{kg} \cdot \mathrm{min})\end{array}$ & 2.1 & 2.6 \\
\hline $\begin{array}{l}\mathrm{AUC}_{\mathrm{GIR}, 0-12 \mathrm{~h}, \mathrm{SS}} / \\
\quad \mathrm{AUC}_{\mathrm{GIR}, \tau, \mathrm{SS}}, \%(\mathrm{CV})\end{array}$ & $53(27)$ & $45(16)$ \\
\hline \multicolumn{3}{|c|}{ Elderly/younger adult $(95 \% \mathrm{CI})$} \\
\hline $\mathrm{AUC}_{\mathrm{GIR}, \tau, \mathrm{SS}}$ & \multicolumn{2}{|c|}{$0.78(0.47-1.31)$} \\
\hline $\mathrm{GIR}_{\max , I D e g, S S}$ & \multicolumn{2}{|c|}{$0.80(0.54-1.17)$} \\
\hline
\end{tabular}

Mean values are estimated least square means, except for the mean ratio of $\mathrm{AUC}_{\mathrm{GIR}, 0-12 \mathrm{~h}, \mathrm{SS}} / \mathrm{AUC}_{\mathrm{GIR}, \tau, \mathrm{SS}}$, which was calculated and summarised using descriptive statistics

$A U C_{G I R, \tau, S S}$ area under the glucose infusion rate (GIR) curve at steady state, $C V$ coefficient of variation, $C I$ confidence interval, GIR $R_{\text {max,- }}$ IDeg,SS maximum GIR at steady state

and treatment period (Period 1/Period 2) as fixed factors. Based on this model, age group means for absolute values were estimated and mean age group ratios were estimated together with two-sided $95 \%$ confidence intervals (CIs). Mean $\mathrm{AUC}_{\mathrm{IDeg}, 0-12 \mathrm{~h}, \mathrm{SS}} / \mathrm{AUC}_{\mathrm{IDeg}, \tau, \mathrm{Ss}}$ and $\mathrm{AUC}_{\mathrm{GIR}, 0-12 \mathrm{~h}, \mathrm{SS}} /$ $\mathrm{AUC}_{\mathrm{GIR}, \tau, \mathrm{SS}}$ were calculated and summarised using descriptive statistics. Safety endpoints were summarised using descriptive statistics.

\section{Results}

\subsection{Subjects}

Forty-two subjects were screened, of whom 14 did not meet the inclusion and/or exclusion criteria, and one withdrew consent. Twenty-seven subjects were randomised to treatment sequences (elderly subjects, $n=14$; younger adults, $n=13$ ), and 26 subjects completed the trial (one subject in the elderly group was withdrawn from the trial because of a serious AE (detailed in Sect. 3.4).

All subjects were Caucasian, and the distribution of male and female subjects and mean BMI in each group was comparable (Table 1). Subjects had a mean [standard deviation (SD)] age of 68 (3) years and 27 (5) years in the elderly and younger adult groups, respectively, and a mean duration of diabetes (SD) of 41 (16) years and 14 (6) years, respectively (Table 1).

\subsection{Steady-State Pharmacokinetics}

Serum concentrations of IDeg were similar in elderly subjects and younger adults, including mean IDeg concentration-time profiles at steady state (Fig. 1).
No statistically significant differences were observed in $\mathrm{AUC}_{\mathrm{IDeg}, \tau, \mathrm{SS}}$ and $C_{\text {max,IDeg,Ss }}$ between elderly subjects and younger adults (Table 2). Estimated mean age group ratios (elderly/younger) for $\mathrm{AUC}_{\mathrm{IDeg}, \tau, \mathrm{SS}}$ and $C_{\mathrm{max}, \mathrm{IDeg}, \mathrm{SS}}$ and corresponding two-sided $95 \%$ CIs were 1.04 (95\% CI 0.73-1.47) and 1.02 (95\% CI 0.74-1.39), respectively. Mean $\mathrm{AUC}_{\mathrm{IDeg}, 0-12 \mathrm{~h}, \mathrm{SS}} / \mathrm{AUC}_{\mathrm{IDeg}, \tau, \mathrm{SS}}$ was $53 \%$ in both elderly subjects and younger adults (Table 2), suggesting that in both age groups IDeg exposure was evenly distributed across the first and second $12 \mathrm{~h}$ of the 24-h dosing interval.

Elderly subjects and younger adults reached clinical steady state after 2-3 days of dosing, based on trough IDeg serum concentrations; thus, concentrations increased only over the first 2-3 days before reaching a plateau. Serum IDeg concentrations at steady state were similar from day to day (data not presented).

\subsection{Steady-State Pharmacodynamics}

No statistically significant differences were observed in $\mathrm{AUC}_{\mathrm{GIR}, \tau, \mathrm{SS}}$ (primary endpoint) and $\mathrm{GIR}_{\text {max,IDeg,SS }}$ between elderly subjects and younger adults (Table 3 ). Estimated mean age group ratios (elderly/younger adult) for $\mathrm{AUC}_{\mathrm{GIR}, \tau, \mathrm{SS}}$ and $\mathrm{GIR}_{\text {max,IDeg,SS }}$ and corresponding twosided $95 \%$ CIs were 0.78 (95\% CI 0.47-1.31) and 0.80 (95\% CI 0.54-1.17), respectively. Mean $\mathrm{AUC}_{\mathrm{GIR}, 0-12 \mathrm{~h}, \mathrm{SS}} /$ $\mathrm{AUC}_{\mathrm{GIR}, \tau, \mathrm{SS}}$ was $53 \%$ in elderly subjects and $45 \%$ in younger subjects (Table 3 ), suggesting that in both age groups the glucose-lowering effect of IDeg was close to evenly distributed across the first and second $12 \mathrm{~h}$ of the 24-h dosing interval.

No subjects were withdrawn because of escape from glucose clamp, and throughout the procedure plasma glucose levels did not deviate considerably in any subject from the clamp target of $5.5 \mathrm{mmol} / \mathrm{L}(100 \mathrm{mg} / \mathrm{dL})$. There was no difference in the coefficient of variation (CV) for the observed glucose levels ( $0-24 \mathrm{~h})$ between elderly and younger adult subjects (CV 8.1 vs. $7.5 \%$ ).

To calculate the duration of action of IDeg, the end of IDeg action was defined as the time at which the plasma glucose level exceeded $8.3 \mathrm{mmol} / \mathrm{L}(150 \mathrm{mg} / \mathrm{dL})$. The plasma glucose level did not exceed $8.3 \mathrm{mmol} / \mathrm{L}$ for the duration of the 26-h clamp procedure in any subject, therefore the duration of action of IDeg was more than $26 \mathrm{~h}$ in all elderly subjects and younger adults (data not presented).

\subsection{Safety}

IDeg was generally well tolerated in this trial. Fifteen treatment-emergent AEs were reported in eight (29.6\%) subjects. Thirteen events occurred in six elderly subjects 
and two events occurred in two younger adult subjects; all but one AE was mild. The most common AEs were headache, with four events occurring in four subjects (three elderly and one younger adult subject), and peripheral oedema, with four events occurring in one elderly subject (this repetitive event largely accounts for the overall apparent disparity in AE numbers between the two age groups). One injection-site reaction, defined as a burning sensation that lasted for approximately $30 \mathrm{~min}$ and that was mild in severity, was reported. A total of 74 treatmentemergent confirmed hypoglycaemic episodes were reported for $23(85.2 \%)$ subjects. Only one severe AE (hypoglycaemic episode) following treatment with IDeg was reported, in the elderly group. This event was considered possibly related to IDeg and the subject was withdrawn from the trial. The overall frequency of hypoglycaemia reported in this trial may be artificially high, because all subjects received a fixed dose of $0.4 \mathrm{U} / \mathrm{kg}$ for 6 consecutive days, irrespective of their individual insulin requirements. Thus, it is likely that the risk of hypoglycaemia was increased for those subjects with a usual total daily basal insulin dose of less than $0.4 \mathrm{U} / \mathrm{kg}$. No clinically significant changes in laboratory parameters, vital signs, physical examination or ECG occurred.

\section{Discussion}

The present study is the first to evaluate the steady-state pharmacokinetic and pharmacodynamic properties of IDeg, using a 26-h euglycaemic clamp procedure, in elderly subjects with type 1 diabetes. We report that the ultra-long pharmacokinetic and pharmacodynamic properties of IDeg observed in younger adults with type 1 diabetes were the same in elderly subjects with type 1 diabetes. Accordingly, the duration of action of IDeg was beyond the clamp duration of $26 \mathrm{~h}$ (end of clamp) in all subjects in both age groups. In an earlier study in subjects with type 1 diabetes, the duration of action was reported to be greater than $42 \mathrm{~h}$ [9].

The exposure level of IDeg at steady state was similar in elderly and younger adult subjects. In both age groups, it took 2-3 days to reach steady-state exposure, and thereafter the total exposure of IDeg was unchanged from day to day. As the pharmacokinetic profiles at steady state were similar between age groups, and the total exposure for the elderly subjects was comparable to that of the younger adult group, this suggests that the absorption properties of IDeg observed in younger adults [5, 6] are preserved in elderly subjects. The glucose-lowering effect of IDeg at steady state was numerically lower (but not significantly different) in elderly subjects versus younger adults, possibly because of the expected decrease in insulin sensitivity with age [10]. Furthermore, the numerical difference in effect is likely to be of limited clinical impact given that insulin is titrated based on individual patient needs. This finding is in concurrence with recent data from a subanalysis of the LIGHT observational study, in which the glucose-lowering effect of long-acting insulin was lower in elderly subjects compared with younger subjects [11].

IDeg was well tolerated in younger and elderly subjects with type 1 diabetes. The incidence of hypoglycaemia in this trial could be considered artificial to some extent. This is because a fixed dose level independent of subjects' individual insulin requirements was applied in this trial, to be able to compare the pharmacokinetic/pharmacodynamic response between younger and elderly subjects.

The strengths of this study include the assessment of both pharmacokinetic and pharmacodynamic properties at steady state in a once-daily dosing regimen, which is the most clinically relevant setting. A 6-day dosing period was used, thereby ensuring that both a pharmacokinetic steady state and stable glycaemic control were achieved before the last dose administered during each treatment period. A further strength of the study is that only subjects with type 1 diabetes were included. This enabled assessment of the pharmacodynamic response in a euglycaemic glucose clamp without interference from the effect of endogenous insulin, which would have been a risk if healthy subjects or subjects with type 2 diabetes had been included. A potential limitation of the study was the relatively low number of subjects included. Large-scale clinical studies to investigate the efficacy and safety of IDeg in the elderly population will be able to allow further sub-analyses evaluating more discrete age ranges.

Although the availability of clinical data regarding the use of long-acting insulins in elderly subjects is limited [2, 12-15], a number of studies have reported efficacy of such products in elderly subjects [14] and decreased rates of hypoglycaemia compared with other treatments [12-16]. Of particular note is a recent meta-analysis comparing hypoglycaemia rates of IDeg with IGlar in elderly subjects with type 1 or type 2 diabetes [16]. In this pooled population, a numerically lower (by $21 \%$ ) rate of overall confirmed hypoglycaemia was reported with IDeg compared with IGlar during the maintenance period, once the optimal dosage had been determined. Furthermore, nocturnal confirmed hypoglycaemia was significantly lower (by $35 \%$ ) with IDeg compared with IGlar [16]. Based on these results, IDeg could be of particular benefit in elderly patients, who may have a higher propensity for hypoglycaemia owing to a higher incidence of co-morbidities and complications associated with long-standing disease.

In conclusion, the results of the present study show that the ultra-long and stable pharmacokinetic and pharmacodynamic properties of IDeg observed in younger adults are 
preserved in elderly subjects. These attributes may explain observations from the earlier meta-analysis in which elderly subjects had lower rates of hypoglycaemic episodes, particularly nocturnal episodes, with IDeg [16]. Thus, IDeg is an effective basal insulin treatment for elderly patients with diabetes.

Acknowledgments This study was supported financially by Novo Nordisk A/S, Denmark, which was also responsible for the design, analysis and reporting of the study, with input from the authors. All authors were involved in the conception and design or analysis and interpretation of data, as well as drafting the article or revising it critically for important intellectual content. The authors thank all the subjects who participated in the study. The authors also thank ApotheCom ScopeMedical, UK, for medical writing support, funded by Novo Nordisk.

Conflict of interest and funding Stefan Korsatko, Sigrid Deller, Julia K. Mader, Gerd Koehler, Gerlies Treiber and Thomas R. Pieber have received travel costs for the presentation of data and/or fees for speaking and/or consulting from Novo Nordisk $\mathrm{A} / \mathrm{S}$ in the previous 3 years. Thomas Pieber is a member of a Novo Nordisk A/S advisory board. Katharina Glettler, Martina Urschitz and Michael Wolf do not have any financial disclosures to make. Hanne Hastrup, Flemming Søndergaard and Hanne Haahr are employees and shareholders of Novo Nordisk A/S. There are no other relationships or activities that could appear to have influenced the submitted work.

Open Access This article is distributed under the terms of the Creative Commons Attribution Noncommercial License which permits any noncommercial use, distribution, and reproduction in any medium, provided the original author(s) and the source are credited.

\section{References}

1. American Diabetes Association. Standards of medical care in diabetes: 2012. Diabetes Care. 2012;35:S11-63.

2. Ligthelm RJ, Kaiser M, Vora J, Yale JF. Insulin use in elderly adults: risk of hypoglycemia and strategies for care. J Am Geriatr Soc. 2012;60:1564-70.

3. Brown AF, Mangione CM, Saliba D, Sarkisian CA. Guidelines for improving the care of the older person with diabetes mellitus. J Am Geriatr Soc. 2003;51:S265-80.

4. Meneilly GS, Tessier D. Diabetes in the elderly. Diabet Med. 1995;12:949-60.

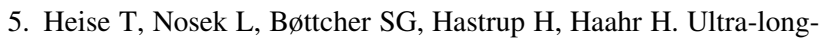
acting insulin degludec has a flat and stable glucose-lowering effect in type 2 diabetes. Diabetes Obes Metab. 2012;14:944-50.

6. Heise T, Hermanski L, Nosek L, Feldman A, Rasmussen S, Haahr $\mathrm{H}$. Insulin degludec: four times lower pharmacodynamic variability than insulin glargine under steady-state conditions in type 1 diabetes. Diabetes Obes Metab. 2012;14:859-64.

7. Frid A, Hirsch L, Gaspar R, Hicks D, Kreugel G, Liersch J, Letondeur C, Sauvanet JP, Tubiana-Rufi N, Strauss K. New injection recommendations for patients with diabetes. Diabetes Metab. 2010;36:S3-18.

8. American Diabetes Association Workgroup on Hypoglycemia. Defining and reporting hypoglycemia in diabetes. Diabetes Care. 2005;28:1245-9.

9. Kurtzhals P, Heise T, Strauss HM, et al. Multi-hexamer formation is the underlying mechanism behind the ultra-long glucose-lowering effect of insulin degludec. Diabetes. 2011;60(Suppl 1A):42LB.

10. Scheen AJ. Diabetes mellitus in the elderly: insulin resistance and/or impaired insulin secretion? Diabetes Metab. 2005;31: 5S27-5S34.

11. Kerlan V, Vergès B, Tawil C, Lahrichi N, Doucet J. Insulin initiation in elderly patients with type 2 diabetes in France: a subpopulation of the LIGHT study. Curr Med Res Opin. 2012;28:503-11.

12. Garber AJ, Clauson P, Pedersen CB, Kølendorf K. Lower risk of hypoglycemia with insulin detemir than with neutral protamine Hagedorn insulin in older persons with type 2 diabetes: a pooled analysis of phase III trials. J Am Geriatr Soc. 2007;55:1735-40.

13. Papa G, Fedele V, Chiavetta A, Lorenti I, Leotta C, Luca S, Rabuazzo AM, Piro S, Alagona C, Spadaro L, Purrello F, Pezzino V. Therapeutic options for elderly diabetic subjects: open label, randomized clinical trial of insulin glargine added to oral antidiabetic drugs versus increased dosage of oral antidiabetic drugs. Acta Diabetol. 2008;45:53-9.

14. Wolffenbuttel BH, Klaff LJ, Bhushan R, Fahrbach JL, Jiang H, Martin S. Initiating insulin therapy in elderly patients with type 2 diabetes: efficacy and safety of lispro mix 25 vs. basal insulin combined with oral glucose-lowering agents. Diabet Med. 2009;26:1147-55.

15. Janka HU, Plewe G, Busch K. Combination of oral antidiabetic agents with basal insulin versus premixed insulin alone in randomized elderly patients with type 2 diabetes mellitus. J Am Geriatr Soc. 2007;55:182-8.

16. Sorli C, Warren M, Oyer D, Mersebach H, Johansen T, Gough SCL. Elderly patients with diabetes experience a lower rate of nocturnal hypoglycaemia with insulin degludec than with insulin glargine: a meta-analysis of phase IIIa trials. Drugs Aging 2013. doi:10.1007/s40266-013-0128-2. 